\title{
L'identité en projets : ville, architecture et patrimoine
}

Analyse de concours à Québec et à Toronto

The ldentity Projects: City, Architecture and Heritage. Analysis of competitions

in Quebec city and Toronto

Imen Ben Jemia

\section{OpenEdition}

Journals

Édition électronique

URL : https://journals.openedition.org/crau/412

DOI : $10.4000 /$ crau.412

ISSN : 2547-5746

Éditeur

Éditions du patrimoine

Édition imprimée

Date de publication : 1 décembre 2014

Pagination : 173-181

ISBN : 978-2-7577-0379-3

ISSN : 1296-4077

Référence électronique

Imen Ben Jemia, "L'identité en projets : ville, architecture et patrimoine », Les Cahiers de la recherche architecturale et urbaine [En ligne], 30/31 | 2014, mis en ligne le 14 septembre 2017, consulté le 07 décembre 2022. URL : http://journals.openedition.org/crau/412; DOI : https://doi.org/10.4000/crau. 412 


\section{L'identité \\ en projets: ville, \\ architecture \\ et patrimoine \\ Analyse de concours \\ à Québec et à Toronto}

\section{L'identité comme concept d'analyse pour l'architecture et la ville}

La question de l'intervention contemporaine sur un bâtiment historique rallie à la fois des considérations sur la pratique architecturale actuelle et des réflexions sur le patrimoine bâti. II s'agit d'un questionnement sur la dualité, apparente du moins, entre la question du patrimoine et celle de la contemporanéité dans un contexte de globalisation 1. D'une part, il existe des débats passionnés sur la conservation du patrimoine local dont le champ ne cesse de s'élargir mais dont les pratiques mènent parfois à la muséification des lieux et figent l'identité. D'autre part, on trouve des partisans d'une architecture nouvelle internationale, parfois provocatrice qui porte la signature de ses concepteurs et entend cristalliser l'identité nouvelle de la ville. Entre ces deux positions, à première vue diamétralement opposées, la réflexion disciplinaire sur l'intervention contemporaine au sein de bâtiment historique évolue sous le poids des idéologies, discours et enjeux ${ }^{2}$.

1. Cette contribution est issue d'une thèse de doctorat en aménagement sous l'intitulé L'Identité en projets: ville, architecture et patrimoine, analyse de concours à Québec et Toronto, menée sous la direction de Jacques Lachapelle au Laboratoire d'étude de l'architecture potentielle (Leap) de l'université de Montréal.

2. «Intervenir sur le monument aujourd'hui ne devrait pas tant conduire à faire au patrimoine les effets de la transformation globale de la société mais être le lieu d'une interrogation de la société elle-même: ce qu'elle regarde, ce qu'elle décide de protéger, à quel point il lui est difficile de conserver des bâtiments sans usage, dont il faut faire commerce. » Dominique Rouillard, Architectures contemporaines et monuments historiques: guide des réalisations en France depuis 1980, Paris, Le Moniteur, 2006, p.15-16. 
Le concept d'identité est suffisamment englobant pour inclure des dimensions larges et duelles, au regard des questions de territorialités et de temporalités. Ainsi, il est approprié tant par les défenseurs du patrimoine et de l'architecture locale que par les protagonistes d'une image contemporaine pour la ville. Le terme identité est en effet, autant intégré à la terminologie référant à l'histoire et à la mémoire, que rattaché au monde de l'innovation, du branding et de la créativité. Par ailleurs, étant employé dans de nombreuses disciplines, il a une grande portée et ne confine pas l'analyse de l'architecture à l'intérieur d'une spécificité disciplinaire qui aurait son propre cadre théorique et ses pratiques. II permet plutôt d'aborder l'architecture sous l'angle d'une contribution à la société, l'influençant et subissant réciproquement son influence ${ }^{3}$. Cette transversalité disciplinaire est d'autant plus nécessaire pour traiter de l'articulation entre architecture et ville que l'identité est rattachée à un territoire et que celui de la ville est déterminant dans un contexte de globalisation.

Notre recherche vise la compréhension des processus de la construction identitaire en relation avec le projet architectural. II est donc principalement question d'un projet identitaire car la définition d'une identité dépend des acteurs qui en parlent. En effet, la littérature qui analyse la question de l'identité dans le monde contemporain y voit une construction sociale ${ }^{4}$. Dans cette perspective, la thèse aborde la dynamique de la mise en place d'une identité architecturale et urbaine comme une opération discursive menée par différents acteurs. Elle traite de la construction identitaire par les pouvoirs publics ainsi que des discours sur l'iden- tification du projet par les architectes. En aval de ce processus, nous observons l'identité produite à travers l'architecture. D'autre part, en aval de ce processus, l'identité produite à travers l'architecture est analysée.

Il est remarquable que le terme identité réfère à la fois à la différenciation et à la ressemblance. En effet, identité semble s'opposer au terme identique car celui-ci implique une ressemblance alors que l'emploi social et politique du terme identité renvoie à une différenciation. Par contre identité et identifié ont la même logique: "La différence, qui constitue l'identité, repose toujours sur ce qui est propre et exclusif à un être; il faut donc que celui-ci soit identifié par un caractère qui le singularise ${ }^{5}$. "

Cette quête des spécificités amène les acteurs à valoriser parfois des caractéristiques propres, et d'autres fois de nouveaux attributs. Au niveau de la ville et de l'architecture, cette dynamique se traduit d'une part par l'attachement au patrimoine local et d'autre part par l'aspiration aux tendances globales. Dans un contexte de globalisation, de plus en plus de villes se dotent d'une identité propre pour se démarquer à l'échelle internationale. La compétition se justifie par les références aux réseaux de villes globales, aux villes du patrimoine mondial, aux villes de design, etc. L'architecture devient un moyen de communication utilisé pour transmettre une identité visuelle.

Ainsi, l'intérêt d'une recherche sur la construction identitaire des villes et la pratique architecturale au sein du patrimoine tient au fait qu'elle navigue entre la compréhension des enjeux identitaires au sein de la société (ou collectivité) et l'interprétation qu'en font
3. L'implication de l'architecture dans la dynamique identitaire a été soulignée par Carmen Popescu: « If architecture was previously connected allusively to identity (a metaphor of the human condition), now it becames explicitly involved in building it. [Si l'architecture a toujours été reliée allusivement à l'identité (une métaphore de la condition humaine), de nos jours elle participe explicitement à la construction identitaire.] » Carmen Popescu, "Space, Time: Identity », National Identities, 2006, 8, 3, p. 189-206. (trad. Imen Ben Jemia)
4. Charles Taylor, «Les sources de l'identité moderne », dans Mikhaël Elbaz, Andrée Fortin, Guy Laforest (dir.), Les Frontières de l'identité, modernité et postmodernité au Québec, Québec, Les Presses de l'université Laval, 1996, p. 347-364.
5. www.larousse.fr/encyclopedie/ nom-commun-nom/ identit\%C3\%A9/59715\#904546 consulté le 6 juillet 2011. 
les concepteurs des projets. D'une part, les choix de la commande témoignent du rapport de la ville avec son histoire, son présent et son futur ainsi que des enjeux qui la caractérisent. D'autre part, les choix des architectes révèlent un processus conceptuel et une identification à des attributs culturels tout en traduisant l'impact des ambitions de la commande publique. En fin de compte, l'identité de l'architecture produite devient le résultat de ces deux processus et constitue un miroir de l'identité locale à un moment précis de l'histoire.

Sous le prisme identitaire, il s'agit donc de mettre en évidence les enjeux et les idéologies qui imprègnent la pratique architecturale contemporaine en contexte patrimonial. En effet, la plupart des recherches sur le concept d'identité en architecture et son rapport avec la culture locale ou globale l'abordent, soit sous l'angle de la construction sociale, politique et idéologique $^{6}$, soit du point de vue disciplinaire théorique ${ }^{7}$. La présente recherche s'attarde à distinguer l'identité comme discours imprégné par une idéologie propre à une époque et impliquant l'architecture, de l'identité comme caractéristique d'une architecture donnée et une réflexion théorique au sein de la discipline. Cette distinction est établie autant dans le cadre théorique que dans I'analyse empirique.

Les concours d'architecture apparaissent comme des supports méthodologiques privilégiés pour mener l'investigation, déterminer le rôle des différents acteurs et apprécier les multiples facettes du projet identitaire aux étapes successives de son développement. Notre étude cherche ainsi à comprendre comment se construit l'identité de la ville et celle de l'architecture contem- poraine au sein du patrimoine lors d'un concours. Plusieurs questions découlent de cette interrogation première: Pourquoi l'identité devient-elle un concept de référence aujourd'hui? À la lumière de différents cadres théoriques, quels sont les concepts qui y sont reliés? Comment une ville construit-elle son identité en rapport avec la culture locale et la culture globale? Quels sont les enjeux et les motivations de son positionnement identitaire? Comment les architectes intègrentils la référence globale et/ou locale dans leur discours et dans la conception du projet? Quelle est l'identité de I'architecture contemporaine des musées ainsi générée et quel est son rapport avec le patrimoine?

Après l'exploration du concept d'étude et la mise en place du cadre méthodologique, il s'agit d'examiner la construction identitaire des villes et d'expliquer ses dynamiques, sa concrétisation et ses objectifs politiques. Cette perspective générale conduira à démontrer que la manipulation des images qui règne sur la culture contemporaine amplifie le rôle des architectes comme concepteurs d'images de marque. Quels que soient les enjeux motivant la construction identitaire d'une ville, l'architecture devient un moyen de communication privilégié à son service. En effet comme le montrent les travaux de Monique Yaari ${ }^{8}$, la dynamique identitaire, son aspect politique et son emphase sur la communication se retrouve autant dans le cas d'une ville focalisant sur l'architecture globale que dans le cas d'une ville qui valorise son patrimoine historique. Les stratégies d'intervention sur le patrimoine démontrent l'impact de ces enjeux sur les pratiques actuelles. Ces interventions révèlent aussi le rapport de la société avec son histoire
6. Voir par exemple: Luc Noppen (dir.), Architecture, forme urbaine et identité collective, Sillery, Québec, Septentrion, 1995. Lucie K. Morisset, Luc Noppen, Denis Saint-Jacques, Ville imaginaire, ville identitaire: échos de Québec, Québec, Nota Bene, 1999. Lucie K. Morisset, Patrick Dieudonné, Jean-François Simon (dir.), Réinventer pays et paysages, Brest, Centre de recherche bretonne et celtique, 2003. Anne-Marie Broudehoux (dir.),

Paysages construits: mémoire, identité, idéologies, Québec, Éditions MultiMondes, 2006. Daniel Le Couëdic, Jean-François Simon (dir.), Construire dans la diversité. Architecture, contextes et identités, Rennes, Presses universitaires de Rennes, 2005.

7. Voir par exemple: Carmen Popescu, "Linking Notions: A reconstructed Perspective of Identity and Architecture", dans Peter Herrle, Erik Wegerhoff (dir.), Architecture and Identity, Berlin, Lit Verlag, 2008, p. 289-298. Chris Abdel, Architecture and Identity. Responses to Cultural and Technological Change, Oxford, Architectural Press, 2000.

8. Monique Yaari, « Identitaire ou générique: la ville comme objet de communication », dans François Loyer (dir.), Ville d'hier, ville d'aujourd'hui en Europe, Paris, Fayard/Éditions du Patrimoine, 2001, p. 379-396. 
à travers les choix mémoriels qu'elle opère lors de la conservation et à travers les images qu'elle construit par les projets d'architecture. L'étude des projets choisis révèle par ailleurs les spécificités caractérisant la conception du musée contemporain.

\section{Le concours comme support de recherche: deux études de cas}

Deux études de cas font l'objet de la recherche. Il s'agit du concours du Centre d'interprétation de la Place-Royale (fig. 1) à Québec et du concours Renaissance ROM (fig. 2), à Toronto, qui consistait en l'agrandissement du Musée royal de I'Ontario (ou Royal Ontario Museum, dit ROM). du concours du Centre d'interprétation de la Place-Royale (fig. 2) à Québec.

Le concours du Centre d'accueil et d'interprétation de la Place-Royale (1996) dans la ville de Québec est un exemple singulier qui a déclenché une vaste polémique révélatrice des enjeux locaux. Les très nombreuses propositions rendues lors de la première étape ont révélé la diversité des points de vue sur l'architecture contemporaine. Ce concours ne peut être compris que dans la

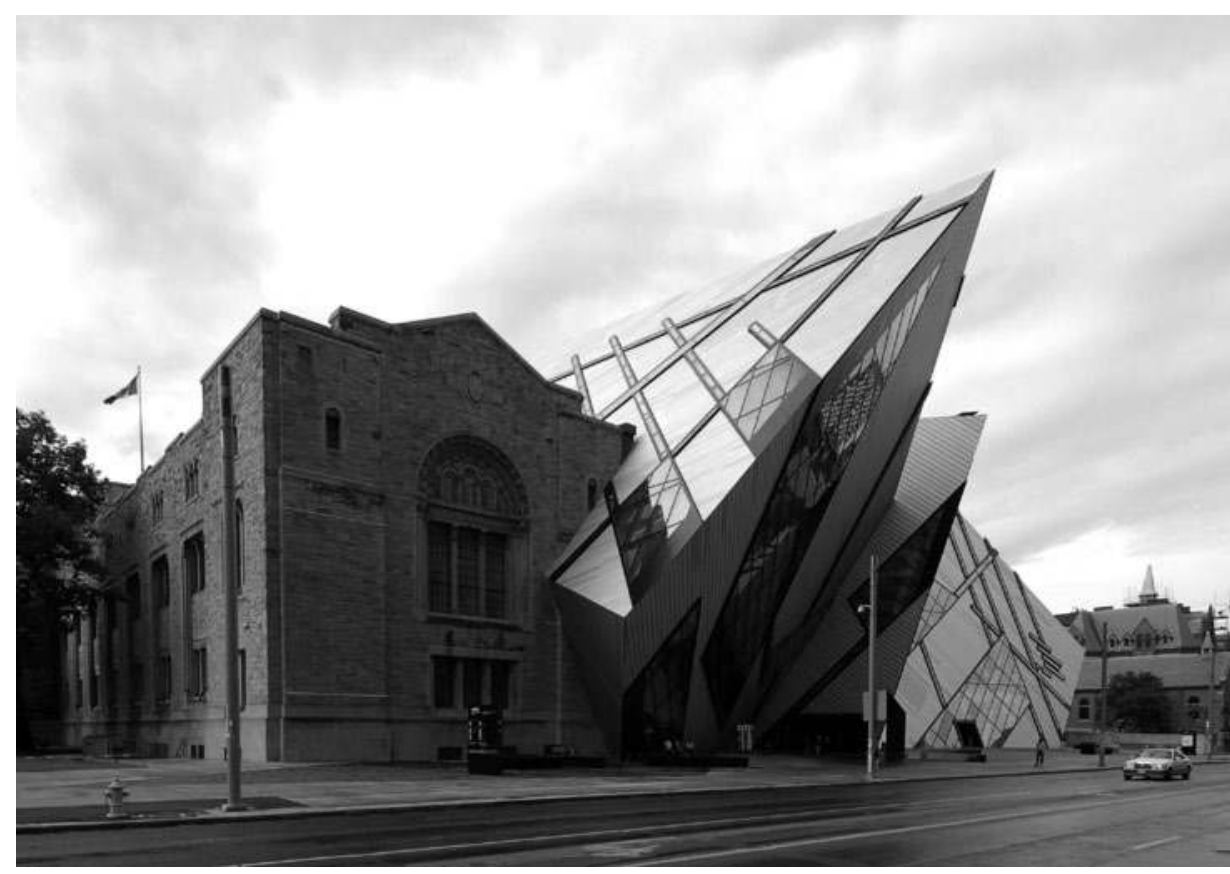

Figure 1: Musée royal d'Ontario, Toronto () Jacques Lachapelle. 
continuité du projet des années 1960-1970 mettant en valeur la basse-ville comme origine de la colonie française. Dans une ville où l'histoire revêt une importance primordiale dans l'affirmation identitaire, le site du projet, qui est une reconstruction pour rendre hommage au passé ${ }^{9}$ est en lui-même révélateur des aspirations historicistes. Alors que le concours visait la concrétisation d'une approche différente de celle du passé, celle-ci a marqué le programme préconisant la conservation de bâtiments existants ainsi que l'interprétation des lieux.

Bien que très différent, d'où son intérêt, le concours Renaissance ROM est aussi un concours éloquent en significations et constitue un autre exemple révélateur de l'apport de l'architecture à la construction identitaire des villes. Son grand impact médiatique informe

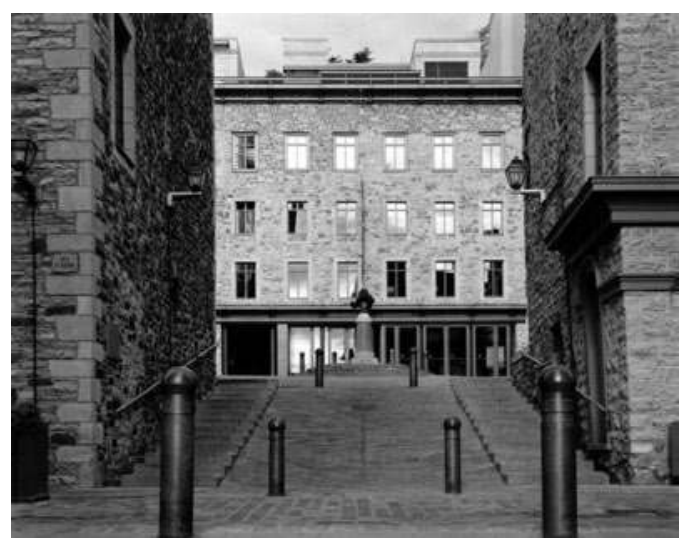

Figure 2: Musée royal de l'Ontario, Toronto.

(C) Jacques Lachapelle. sur le potentiel de l'architecture dans la création d'images. Le Musée royal de l'Ontario, ROM, est une propriété du gouvernement de l'Ontario et fait partie du répertoire des monuments historiques de la ville de Toronto. Le récent concours visant son agrandissement a été lancé en 2001 pour répondre aux nouveaux besoins du musée. Ouvert à l'international, il matérialise la volonté de l'institution de transformer son image et de l'inscrire dans le courant architectural international que l'on observe dans plusieurs musées à travers le monde. Ce projet rejoint ainsi les objectifs de la ville qui ambitionne de se démarquer par ses productions culturelles contemporaines.

À première vue, les contextes ainsi que les projets peuvent sembler très éloignés: d'un côté, un concours à grande échelle, organisé dans une ville internationale et aboutissant à un projet emblématique conçu par un starchitecte ${ }^{10}$, de l'autre côté, un concours à l'échelle nationale, dans une ville du patrimoine mondial et aboutissant à un projet de mise en valeur des vestiges historiques. Pourtant, les deux contextes ont un certain nombre de points communs. Les deux ont des liens avec des réseaux internationaux et ambitionnent une visibilité internationale, le réseau des villes globales et la mode de la starchitecture dans un cas et l'inscription au patrimoine mondial de I'UNESCO dans l'autre. Les programmes des deux concours, de type culturel, sont similaires. Musée et centre d'interprétation font valoir des objets auxquels on confère un statut d'exception: une collection d'œuvres pour l'un, le quartier environnant pour l'autre. Les deux cas impliquent une intervention sur le patrimoine dans deux capitales provinciales canadiennes dans l'objectif
9. Voir à ce sujet: Isabelle Faure, La conservation et la restauration du patrimoine bâti au Québec: étude des fondements culturels et idéologiques à travers l'exemple du projet de Place Royale, Thèse de doctorat, Université de Paris VIII, 1995.
10. Terme qui désigne les architectes de renommée internationale dont les interventions et l'architecture font l'objet d'une grande médiatisation. 
de renforcer le prestige de chacune. De plus, les deux villes sont motivées par des enjeux touristiques. Enfin, dans les deux cas, il s'agit à la fois d'une architecture nouvelle et d'un cadre bâti patrimonial.

Par ailleurs, notre nouvelle approche du concept d'identité fournit un cadre théorique qui permet d'éclairer les liens entre les deux interventions. Les acteurs, les discours, le processus du concours ainsi que les propositions architecturales développées participent en effet aux projets identitaires des deux villes et sont symptomatiques d'une nouvelle condition contemporaine. La focalisation sur l'image et la communication amène une nouvelle réalité de la ville, de la pratique architecturale et de la pratique patrimoniale.

L'analyse des concours d'architecture offre l'avantage d'aborder la construction identitaire tout au long de l'élaboration du projet (fig.3):

Figure 3: Analyse

d'un concours.

\section{Analyse du concours}

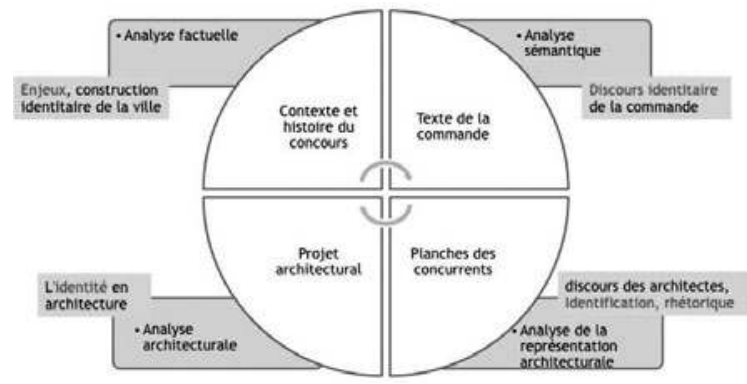

1. En amont, les enjeux politiques et économiques que revêt chaque projet tant pour la ville que pour l'institution, trouvent leur expression dans le programme du concours.

2. Les propositions architecturales permettent d'analyser l'interprétation du programme par les architectes et les référents identitaires qu'ils exploitent. Les différents projets rendus constituent en effet une masse documentaire pour questionner la variété des solutions face à une même commande témoignant des différentes formes de la transcription de l'identité projetée de la ville dans la pratique architecturale.

3. La réception au sein de la société civile est perceptible à travers les publications autour du concours lorsque les documents sont disponibles.

4. Tout au long du processus, le concours devient ainsi un générateur de discours sous forme de publications et de projets, et à travers des textes, des images et une architecture, il constitue en ce sens un support privilégié pour l'appréhension et la compréhension du phénomène de construction identitaire dans les villes contemporaines. L'analyse de ces différents documents révèle la complexité des enjeux identitaires et leurs impacts sur la pratique architecturale.

Étudier deux cas n'implique pas une recherche comparative. II s'agit d'une part, d'illustrer l'usage et les pratiques relatifs aux deux versants, local et global, de la terminologie identitaire. D'autre part, ils permettent une forme de validation de la méthodologie dans deux situations spécifiques. L'étude de cas transite entre la théorie et la recherche empirique. Ce dernier volet prédomine. En effet, l'étude a commencé de manière 
déductive. Elle est fondée sur des concepts existants tels que l'identité et la construction identitaire dans les villes ce qui a permis de préciser progressivement la question de recherche. Toutefois, cette recherche demeure exploratoire particulièrement durant les phases d'analyse des données tout en s'enrichissant de références théoriques utiles à la compréhension des enjeux identitaires.

\section{Le chantier identitaire entre discours et pratiques}

Malgré leurs différences, les deux études de cas ont montré grâce aux analyses factuelles, historiques et de discours, la dynamique du projet identitaire des villes aujourd'hui, son aspect politique et l'emphase sur la communication qui le caractérise. L'analyse de la réception de chaque concours a permis de soulever la polémique qui a entouré les projets réalisés. Cette polémique nous instruit sur l'écart qui subsiste entre la construction identitaire parla commande, I'interprétation de l'identité par les architectes et son appropriation par les citoyens à travers quelques prises de position dans les publications.

Concernant le cas de Québec, une ville attachée à ses spécificités locales, I'analyse du contexte et des discours démontre l'importance du patrimoine et de I'histoire dans la construction de l'identité et la prédominance des enjeux touristiques. Tel que dénoncé par Françoise Choay ${ }^{11}$ dans son ouvrage L'allégorie du patrimoine, l'identité construite de la ville prend la forme d'un miroir où elle contemple son passé, tente de le transmettre, mais surtout le met en scène pour le touriste contemporain. La polémique entourant le projet a révélé la dualité entre le vécu des lieux désirés par les citoyens et l'image touristique proposée aux visiteurs.

Dans le cas de Toronto qui s'active à construire une image de ville globale à l'échelle internationale, un processus de construction d'une identité basée sur l'innovation, similaire à l'effet Bilbao, a été mis en évidence. On peut l'inscrire dans la lignée des travaux de Muriel Rosemberg ${ }^{12}$, de Berci Florian ${ }^{13}$, Hans Mommaas ${ }^{14}$ et Michael Speaks ${ }^{15}$ qui traitent du city branding en rapport avec l'architecture contemporaine et de la théorie du brandscape élaborée par Anna Klingmann ${ }^{16}$, mettant en avant le rôle de l'architecture comme brand. L'analyse a révélé l'aspect branding et événementiel du projet Renaissance ROM où la question patrimoniale est presque évacuée, malgré l'intérêt historique du bâtiment. Elle démontre aussi l'impact des enjeux économiques et touristiques sur le façonnement de I'identité du projet, ainsi que l'influence du contexte socio-politique. La polémique qui a accompagné la mise en place du projet et sa construction témoigne de la sensibilité de la question identitaire de la ville.

L'analyse de l'architecture et de l'identité dans les deux études de cas a également mis en valeur l'importance de la communication au niveau des discours des architectes en révélant l'emphase sur la rhétorique verbale et visuelle. Les résultats de la recherche confirment les prémisses de cette dynamique et ses stratégies reliées au monde de la communication annoncées par des auteurs tels que Elisabeth Tostrup ${ }^{17}$ et Jacques Guillerme ${ }^{18}$. Les analyses ont démontré l'usage de différentes stratégies qui misent parfois sur la synthèse
11. Françoise Choay, L'allégorie du patrimoine, Paris, Seuil, 1999

12. Muriel Rosemberg, Le marketing urbain en question: production d'espace et de discours dans quatre projets de villes, Paris, Anthropos, diffusion Economica, 2000.

13. Berci Florian, "The city as a brand Orchestrating a unique experience ", dans Véronique Patteeuw (dir.), City Branding: Image Building \& Building Images, Rotterdam, Nai Uitgers, 2002, 20-31.
14. Hans Mommaas, "City Branding. The necessity of socio-cultural goals », dans ibid., p. 34-44

15. Michael Speaks, «Individualization without identity », dans ibid., p. 50-65.

16. Anna Klingmann, Brandscapes: Architecture in the Experience Economy, Cambridge (MA), MIT Press, 2007.
17. Elisabeth Tostrup, Architecture and Rhetoric. Text and Design in Architectural Competitions, Oslo 1939-1997, Londres, Andreas Papadakis Publisher, 1999.

18. Jacques Guillerme, "L'ingestion du rendu », AMC Architecture Mouvement Continuité, «Architecture et Publicité », n॰ 35, 1974, p. 29-32. 
du projet en image choc et d'autres fois sur l'argumentatif et la narration. Ainsi, par exemple dans le cas du concours du Centre d'interprétation de la Place-Royale, l'agence Cimaise utilise un texte narratif, sur le thème de "la recherche du temps perdu», composé de différentes fictions pour mettre en scène son discours. Les choix énonciatifs appuient les choix iconographiques et confortent une mise en scène d'un récit qui appelle à la nostalgie. (fig. 4)
L'analyse des projets soumis pour le concours du Centre d'interprétation de la Place-Royale montre que la référence au local est manifeste dans le processus de la conception. Cette référence est parallèle au discours de la commande qui mise fortement sur une identité locale de la ville. D'une part, l'analyse des discours textuels et visuels a montré la présence de la ville dans les discours des architectes et les différentes formes que cette présence prend. Cet imaginaire de la ville s'appuie sur

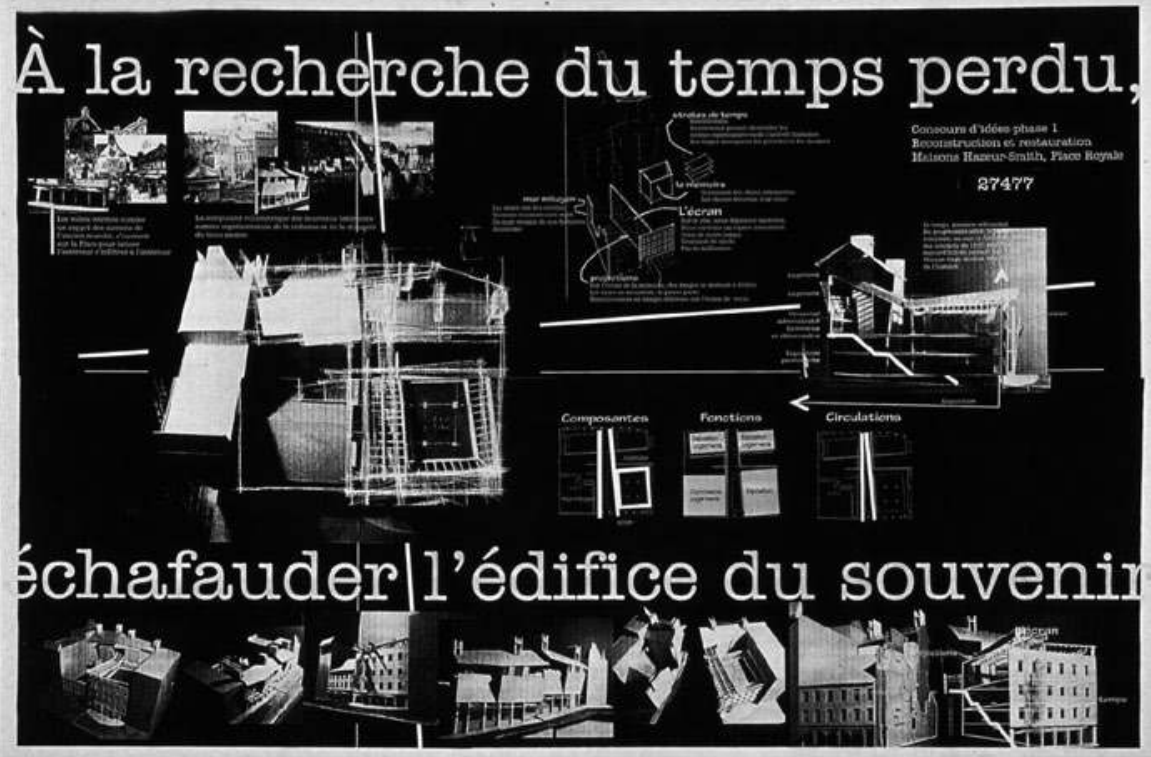

Figure 4: Planche de la première étape du concours du Centre d'interprétation de la Place-Royale, Cimaise. Source Leap. 
le territoire local qui réfère autant à des lieux, éléments et monuments historiques, à des éléments physiques et topographiques qu'au patrimoine immatériel et à I'histoire des lieux. D'autre part, l'analyse architecturale montre les différentes stratégies d'intégration de la composante historique dans l'identité du projet.

Les projets soumis pour le concours Renaissance ROM, où la commande mise sur l'architecture globale pour construire l'identité de la ville, ont multiplié les références aux icônes locales et internationales, et ont focalisé sur l'attractivité. Ces stratégies misant sur la monumentalité et l'iconicité rejoignent la tendance internationale de l'architecture globale présentée dans le cadre théorique. L'exemple du croquis de la firme l'agence Bing Thom révèle une volonté de produire une image choc résumant le projet à ses composantes emblématiques: dans un dessin usant de l'effet esquisse, la ligne directrice du projet, issue du skyline de la ville est aussi associée avec l'image d'un plésiosaure en référence à la collection en histoire du musée. (fig. 5) Ces projets présentent aussi des éléments témoignant du processus de conception d'un musée contemporain. Ainsi, les architectes s'inspirent de références atemporelles et universelles, et les projets montrent l'importance de l'aspect expérientiel des espaces conçus et l'importance de leur relation avec l'environnement urbain. Parmi les différentes soumissions pour ce concours qui a été instrumentalisé pour la communication, c'est le meilleur communicateur qui a gagné au détriment d'architectes plus investis dans l'élaboration de solutions proprement architecturales.

Les projets des deux concours révèlent aussi une différence de la pratique au sein du patrimoine, qui

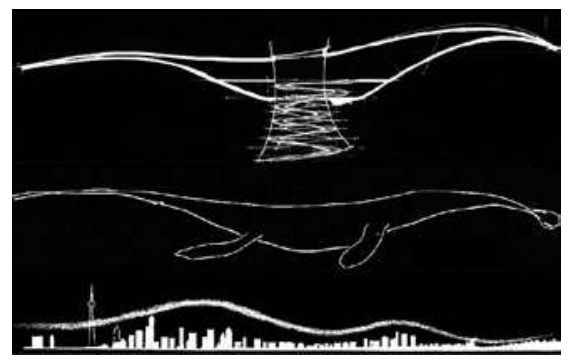

Figure 5:

Esquisses pour le concours

Renaissance ROM, présentation finale. (c) Bing Thom. dépend des contextes et de la commande. Quand I'histoire est sacralisée, il y a une mise à distance et une spectacularisation du patrimoine. Dans le cas où l'emphase est plutôt centrée sur l'innovation, il y a une attraction visuelle du nouveau bâtiment qui est parallèle à l'inclusion et une intégration de l'objet patrimonial au profit d'un espace unifié. Le patrimoine devient un élément composant le projet, sans mise en scène particulière et sans s'accompagner d'une rhétorique discursive identitaire.

Ainsi, les deux études de cas ont démontré les différentes facettes de la construction identitaire et ont dévoilé, à travers l'analyse des discours et des pratiques, les enjeux spécifiques à chaque ville d'une part et communs à la société contemporaine d'autre part. Au-delà de la tension entre le nouveau et l'ancien, entre le patrimoine et la culture globale, la question consiste en la définition même de l'architecture au sein des villes en rapport avec la construction identitaire. Le paradigme régnant, amplifiant l'importance des communications visuelles, transforme parfois les villes en musées à ciel ouvert, produits touristiques destinés au développement économique. Entre patrimonialisation et spectacularisation, la spécificité identitaire, qu'elle soit rattachée à la culture locale ou globale, devient un enjeu politique et une plus-value économique qui sert les besoins de différenciation et de visibilité des villes au niveau international. 